Archive for

Organic Chemistry
Arkivoc 2018, part vii, 0-0 to be inserted by editorial office

\title{
Synthesis of novel C-2 substituted imidazoline derivatives having the norbornene/dibenzobarrelene skeletons
}

\author{
İrfan Çapan ${ }^{a}$ and Süleyman Servi ${ }^{* b}$ \\ ${ }^{a}$ Gazi University, Technical Sciences Vocational College, Department of Polymer Technology, 06560 Ankara, \\ Turkey \\ ${ }^{* b}$ Fırat University, Faculty of Science, Department of Chemistry, 23169 Elazığ, Turkey \\ E-mail: sservi@firat.edu.tr
}

\section{Abstract}

Novel imidazoline derivatives were synthesized from the norbornene and dibenzobarrelene skeletons which were obtained by the Diels-Alder reactions of anthracene and cyclopentadiene with the different dienophiles, such as fumaronitrile and fumaric acid. Synthesis of the C-2 substituted imidazolines was performed with high yields from various dinitriles and diacyl dichlorides.
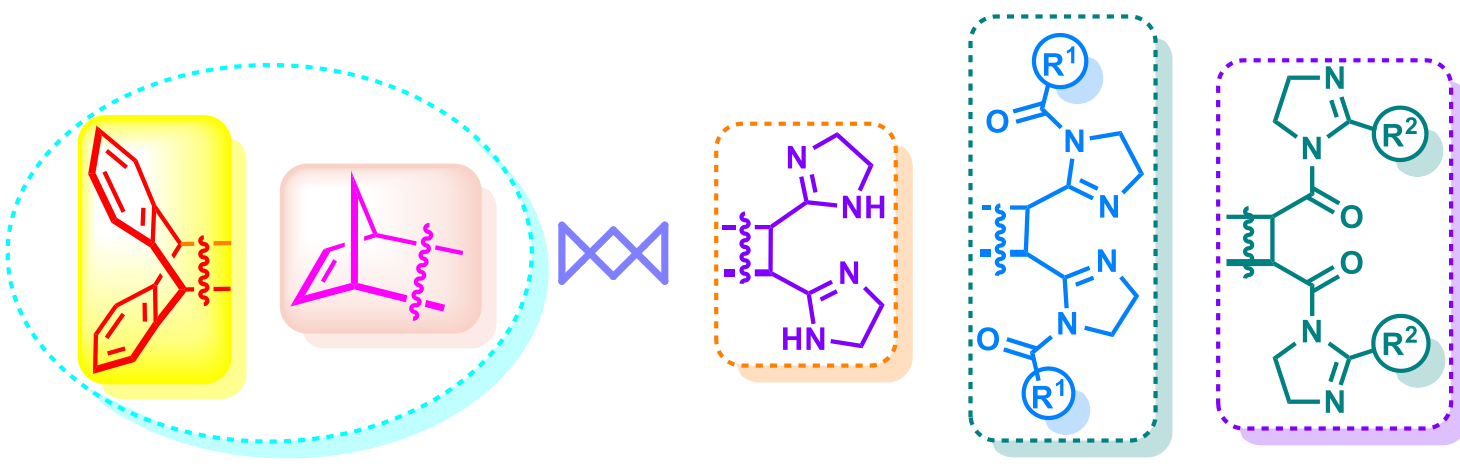

Keywords: Cycloadditions, imidazoline, norbornene, dibenzobarrelene, guanidine, amide 


\section{Introduction}

The chemistry of heterocyclic compounds is one of the most complex and important branches of organic chemistry. The imidazoline ring is an important member of the class of the diaza-heterocyclic compounds. Since natural or synthetic originated imidazolines are found in the molecular structure of many drugs as biologically active residues, imidazoline compounds are extensively used in medicinal and pharmaceutical chemistry. These compounds have widely attracted the focus of biologists because of their potent properties such as anti-inflammatory, antitumor, and antiviral activities. ${ }^{1}$ Imidazoline derivatives, like topsentin B1, D, cylindrospermopsin, and spongotine $\mathrm{A}-\mathrm{C}$ have been isolated from several sea sponges and numerous studies have been carried out on the artificial synthesis and biological activities of these marine compounds. ${ }^{2}$

The synthesis of C-2 substituted imidazolines such as nutlin compounds is of great importance in terms of both organic and medicinal chemistry because nutlin compounds are known to exhibit anti-tumor activity. Substitution at $\mathrm{C}-2$ of the imidazoline ring system is a requirement for anti-tumor activity. ${ }^{3}$ Since the discovery of the imidazoline receptor, many biologically active 2 -imidazoline derivatives have been synthesized. ${ }^{4}$ Clonidine, moxonidine, and phentolamine, which are the most well-known imidazoline receptors, are widely used in the treatment of the diseases such as heart diseases and high blood pressure.

There are quite a number of synthetic procedures developed using different starting materials for the synthesis of C-2 substituted imidazoline derivatives and some of them have been used for more than half a century. ${ }^{5}$ In the past two decades, imidazoline synthetic methods have been modified and optimized for better yields. In addition to classical methods, ${ }^{5,6}$ different procedures such as on a solid support, ${ }^{7}$ solventfree, ${ }^{8}$ sonochemical ${ }^{9}$ and microwave-assisted methods are used in the synthesis of these compounds. ${ }^{10,11}$ Besides, effective and new enantioselective methods have been improved for the synthesis of chiral imidazoline compounds over the last years. ${ }^{12,13}$

Imidazoline compounds are widely studied by organic chemists with regard to their different application areas as synthetic intermediates, protecting groups, chiral auxiliaries and catalysts for asymmetric synthesis. In recent years, they have gained importance as organocatalysts ${ }^{14-16}$ in very diverse stereoselective reactions due to their basicity and nucleophilicity ${ }^{17}$ as well as Brønsted acidity ${ }^{18,19}$ of their salts.

It has been determined that some compounds having the dibenzobarrelene skeleton show a high fluorescence property at room temperature..$^{20}$ Compounds which contain heterocyclic ring fused to the norbornene skeleton, ${ }^{21}$ anthracene-9-imidazoline hydrazone, and its copper (II) complex are known to have antitumor and cytotoxic effects. ${ }^{22}$

There are no imidazoline derivatives associated with norbornene and dibenzobarrelene skeletons in the literature. In this respect, novel $\mathrm{C}-2$ and $\mathrm{N}$-acyl substituted imidazolines showing dibenzobarrelene or norbornene moieties were synthesized in this study for the first time.

\section{Result and Discussion}

In the first part of the work, Diels-Alder cycloaddition reaction was used to synthesize the starting reactants from the various dienophiles and dienes. In these reactions, while anthracene and cyclopentadiene were utilized as dienes, fumaronitrile and fumaric acid were employed as dienophiles. As the starting reactants, dinitriles 1 and 2 (Scheme 1), dicarboxylic acids 13, 17 and 26, and their acyl chlorides 14, 18 and 27 (Schemes 3-5), which have norbornene and dibenzobarrelene skeletons, were synthesized on the basis of cited literature. ${ }^{23-26}$ 
There are many various methods in the literature ${ }^{27-30}$ for the synthesis of 2-imidazoline derivatives from the compounds having a nitrile functional group. When these methods are analyzed, two different synthetic strategies come to the forefront. In the first one, the formation of the 2-imidazoline ring performs with twostep reaction from the imidic ester intermediate, which is also known as the Pinner method. ${ }^{27,28}$ In the second one, it proceeds with a one-step reaction using the catalyst. ${ }^{10}$

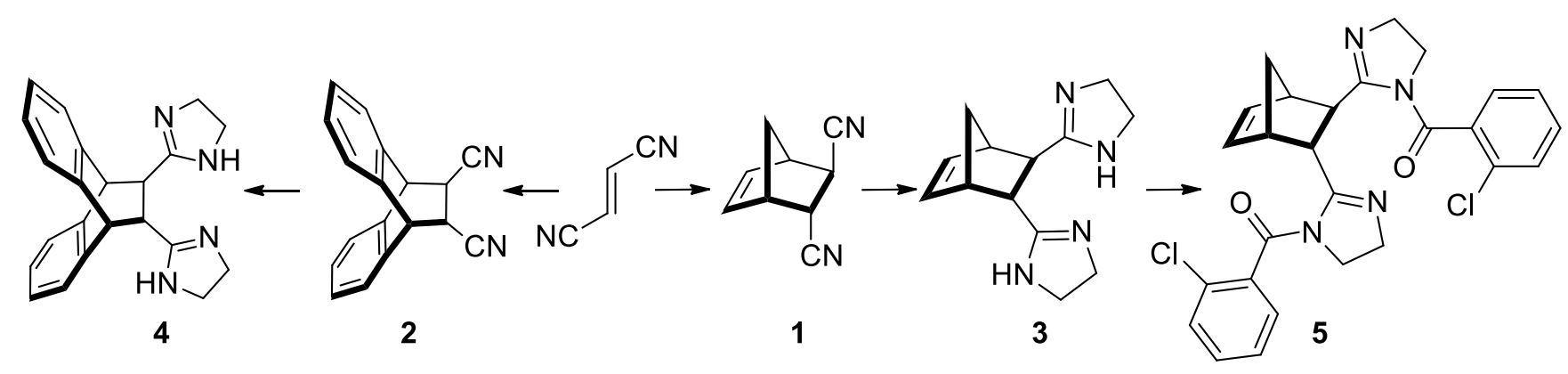

Scheme 1. Synthesis of imidazoline derivatives from the dinitriles having norbornene and dibenzobarrelene skeletons.

Unlike the literature, syntheses of compounds $\mathbf{3}$ and $\mathbf{4}$ were carried out through both conventional and microwave-assisted methods in the solvent-free conditions with high yields (Scheme 1). The reaction time, which is quite long in the conventional method, is rather short in the microwave-assisted procedure. Compound $\mathbf{3}$ was converted to the $\mathrm{N}$-acyl derivative $\mathbf{5}$ by the reaction with 2-chlorobenzoyl chloride in basic medium. In the ${ }^{1} \mathrm{H}$ NMR spectrum of compounds $\mathbf{3}$ and $\mathbf{4}$, while the imidazoline ring protons signals appeared at 3.0-4.0 ppm, the imidazoline methylene carbon signals were not observed in the APT ${ }^{13} \mathrm{C}$ NMR spectrum. However, when the APT ${ }^{13} \mathrm{C}$ NMR spectrum was taken over a very long time using $\mathrm{CDCl}_{3}$, these signals were observed very weakly. To clarify this situation, $\mathrm{N}$-acyl derivative of compound $\mathbf{3}$ was prepared from the reaction of 2-chlorobenzoyl chloride with compound $\mathbf{3}$. In the APT ${ }^{13} \mathrm{C}$ NMR spectrum of compound $\mathbf{5}$, four different $\mathrm{CH}_{2}$ carbon signals belonging to imidazoline ring were clearly observed at 48.3, 49.1 and-51.1 ppm.

To use in subsequent reactions, aromatic and heteroaromatic substituted imidazolines 6-10 were synthesized from the reaction of the requisite aldehydes with ethylenediamine and $\mathrm{N}$-bromosuccinimide (NBS) in basic medium (Scheme 2). ${ }^{31,32}$ Compounds 6-9 are reported in the literature; ${ }^{33-35}$ however, compound $\mathbf{1 0}$ was synthesized for the first time in this work. Compounds $\mathbf{1 1}$ and $\mathbf{1 2}$ are commercially available chemicals such as 6-9.<smiles>[R]C([CH2+])=O</smiles>

6-12

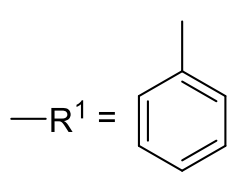

6

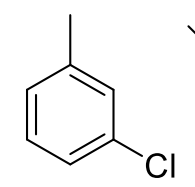

7

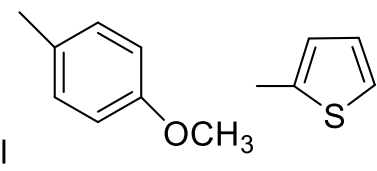

9<smiles>Cc1ccsc1</smiles>

10
$-\mathrm{SH} \quad-\mathrm{SCH}_{3} . \mathrm{HI}$

11

12

Scheme 2. Synthesis of aromatic and heteroaromatic substituted imidazolines 6-10. 
When the ${ }^{1} \mathrm{H}$ NMR spectra of compounds 6-10 were analyzed, the methylene protons belonging to the imidazoline ring were observed as a singlet. ${ }^{31-34}$ In the APT ${ }^{13} \mathrm{C}$ NMR spectrum of compounds 9 and 10 , the methylene carbon signals were observed very poorly. The similar problem was observed in the ${ }^{1} \mathrm{H}$ and ${ }^{13} \mathrm{C}$ NMR spectra of compound 3. The X-ray image of compound $\mathbf{1 0}$ was taken and the doubts about the structural analysis in NMR spectra were eliminated. (Details of the NMR analysis and experimental procedure of compounds 6-10 can be found in the Supporting Information section.)

Compounds 15 and 16 were synthesized through the reaction of acyl chloride 14 with required 2imidazolines 10 and 7 (Scheme 3). The molecular structures of these compounds were characterized with HETCOR NMR spectra, as well as FT-IR, ${ }^{1} \mathrm{H}$ NMR, and APT ${ }^{13} \mathrm{C}$ NMR spectra. In the APT ${ }^{13} \mathrm{C}$ NMR spectra, two different methylene carbon signals belonging to imidazoline ring appeared without observing the situation mentioned above.

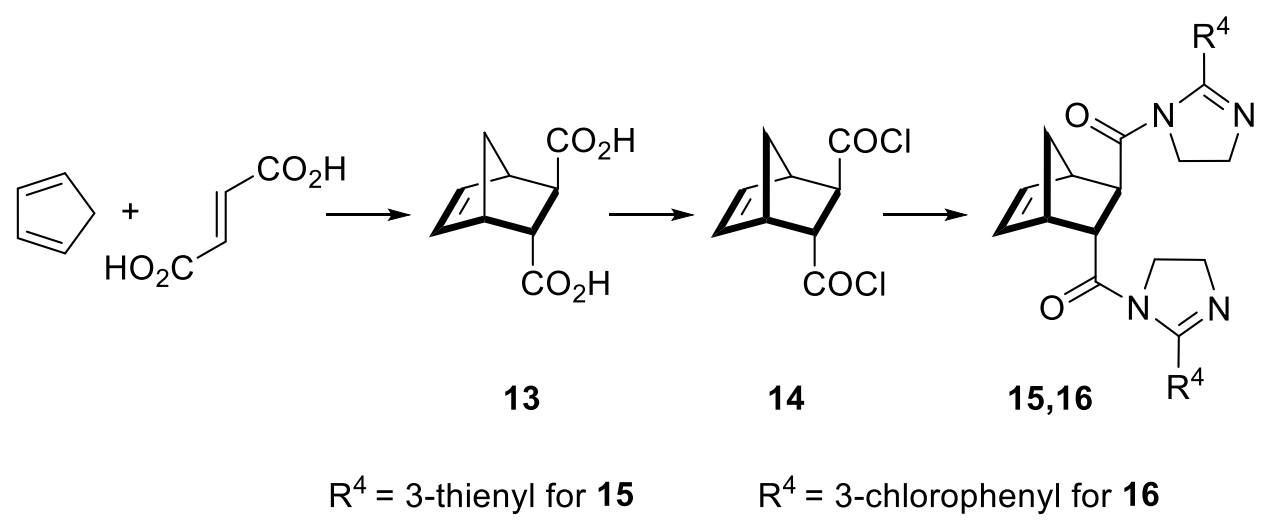

Scheme 3. Synthesis of compounds $\mathbf{1 5}$ and $\mathbf{1 6}$ from acyl chloride $\mathbf{1 4}$ having the norbornene skeleton.

Compound 18 was synthesized by reacting $\mathrm{SOCl}_{2}$ with the diacid 17, which was obtained from the cycloaddition reaction of anthracene and fumaric acid (Scheme 4). Similarly, the synthesis of compound $\mathbf{2 7}$ was performed by hydrolysis of the diester $\mathbf{2 5}$ obtained from the reaction of anthracene with dimethyl acetylenedicarboxylate (DMAD) followed by the reaction of the obtained diacid 26 with $\mathrm{SOCl}_{2}$ (Scheme 5). Compounds 14, 18 and 27 were synthesized in our previous work. ${ }^{23}$ The various $\mathrm{N}$-acyl C-2 substituted imidazoline derivatives 19-24 and 28-31 were prepared through an easy and simple nucleophilic substitution of the aromatic/heteroaromatic imidazolines with the two different acyl chlorides $\mathbf{1 8}$ and $\mathbf{2 7}$ having the dibenzobarrelene skeleton (Schemes 4 and 5).

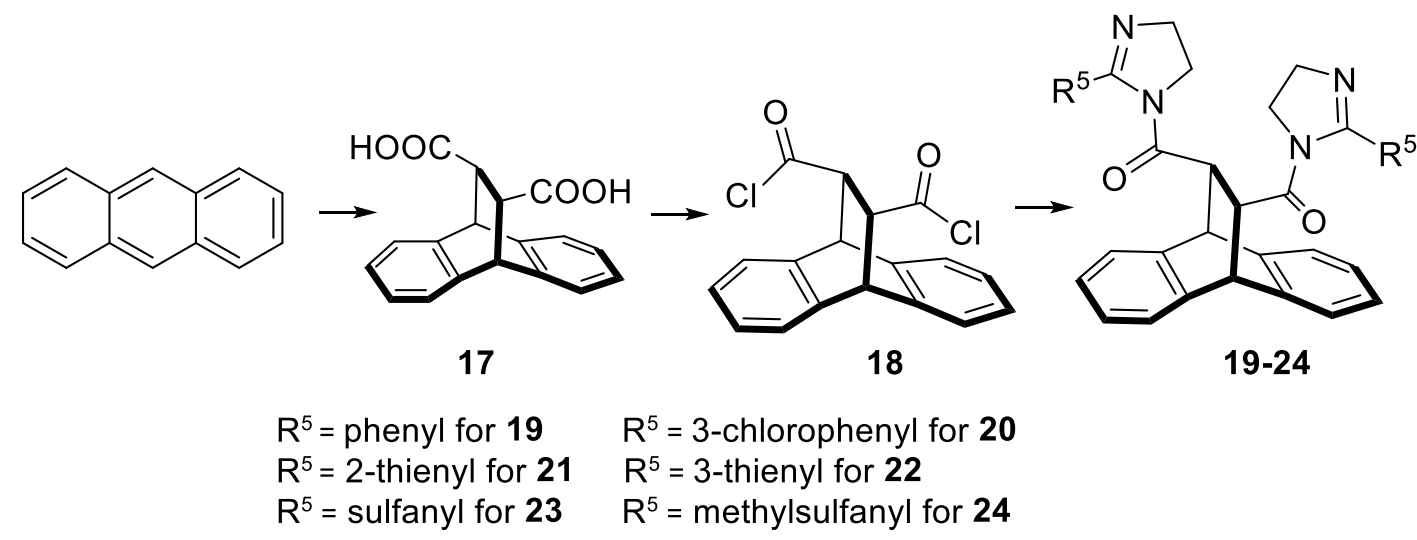

Scheme 4. Synthesis of compounds 19-24 from acyl chloride 18 having the dibenzobarrelene skeleton. 
In the FT-IR spectra, carbonyl stretching vibrations belonging to acyl chlorides $\mathbf{1 8}$ and $\mathbf{2 7}$ were observed at about 1789 and $1783 \mathrm{~cm}^{-1}$, while in the spectra of compounds 19-24 and 28-31, the signal belonging to amide carbonyl appeared at $1680 \mathrm{~cm}^{-1}$. In the ${ }^{1} \mathrm{H}$ NMR spectra of the compounds $6-10$, the methylene protons of the imidazoline ring were observed as a singlet at 3.60 to $3.80 \mathrm{ppm}$, while the methylene protons belonging to the imidazoline ring of the compounds 19-24, which has two protons equivalent to each other, appeared as four different triplets at 3.30-4.30 ppm. Unlike compounds 19-24, in the ${ }^{1} \mathrm{H}$ NMR spectrum of compounds 28-31, two of the four different methylene groups which has four protons equivalent to each other were observed at 3.30-3.60 ppm, while the other two methylene group protons appeared at lower fields (3.8 ppm, equivalent to four protons) as a singlet. In the APT ${ }^{13} \mathrm{C}$ NMR spectra of the compounds 19-24, the methylene carbons belonging to the 2-imidazoline ring were seen at 48 and 54 ppm as two different methylene carbons, as expected. However, these methylene carbons of the compounds 28-31 appeared at about 45-47 ppm at higher fields than the other two methylene carbons. These findings were also supported by HETCOR NMR spectra.

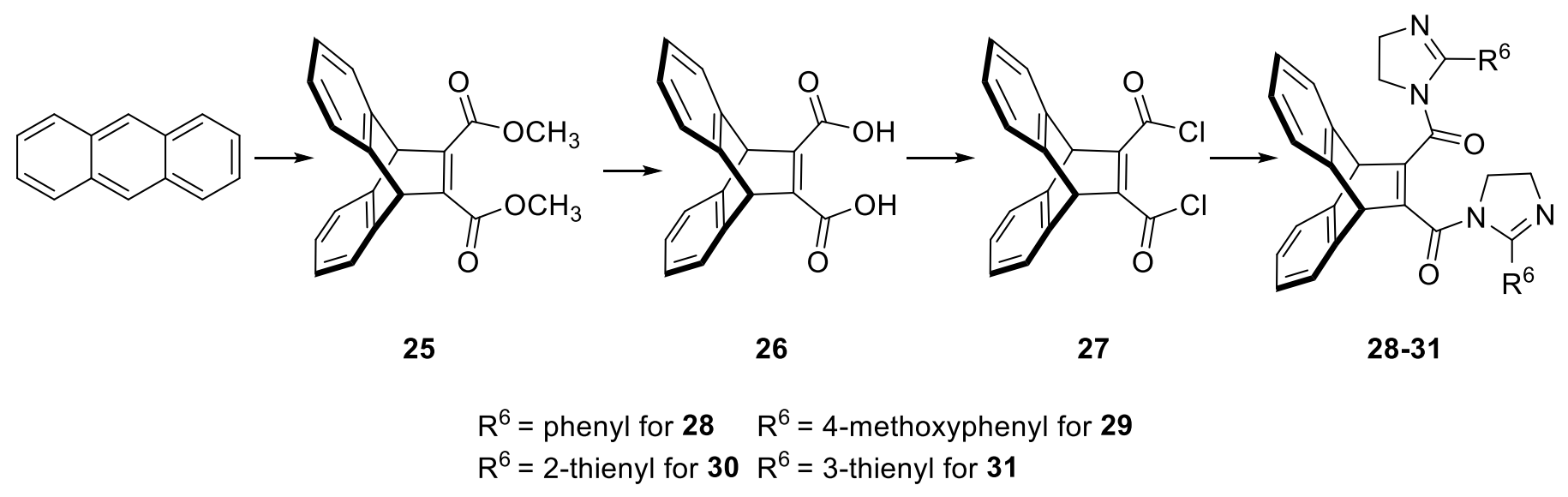

Scheme 5. Synthesis of compounds 28-31 from acyl chloride $\mathbf{2 7}$ having the dibenzobarrelene skeleton.

\section{Conclusions}

In this work, C-2 substituted imidazoline derivatives 3 and $\mathbf{4}$ (Scheme 1) were synthesized from dinitrile compounds, having norbornene and dibenzobarrelene skeletons, in both conventional heating and microwave-assisted methods. These syntheses were carried out under neat reaction conditions unlike the methods in the literature. In addition, novel C-2 and $N$-substituted imidazolines 15, 16, 19-24, 28-31 which contain aromatic, alicyclic, and heterocyclic structures (Schemes 3-5) were obtained in high yields starting from different diacyl dichlorides.

\section{Experimental Section}

General. Reagents were of commercial quality, and all solvents were used after purification. Infrared (IR) spectra were determined on a Perkin-Elmer Spectrum One Fourier transform-infrared (FT-IR) spectrometer. NMR spectra were recorded on Bruker $400 \mathrm{MHz}$ spectrometer. Chemical shift $\delta$ is reported in parts per million relatives to $\mathrm{CHCl}_{3}\left({ }^{1} \mathrm{H} \delta 7.27\right), \mathrm{CDCl}_{3}\left({ }^{13} \mathrm{C} \delta\right.$ 77.0). X-ray analyses were carried out at Gebze Technical University. 
The $\mathrm{C}, \mathrm{H}, \mathrm{N}$ and $\mathrm{S}$ elemental analyses were executed using Leco-932 CHNS/O Elemental Analyzer at the Faculty of Arts and Sciences, University of Bozok, Yozgat, Turkey. The microwave-assisted reactions were performed with single mode CEM Discover Labmate instrument (producing continuous irradiation at $2450 \mathrm{MHz}$ ), which has an in situ magnetic stirrer, irradiation monitored by PC computer, IR measurement, and continuous feedback temperature control. Thin layer chromatography was carried out on precoated $0.2 \mathrm{~mm} \mathrm{Merck}$ Kieselgel 60 F254 plates. Melting points were determined with an Electrothermal IA 9100 apparatus.

The synthetic method and detailed spectral data of trans-bicyclo[2.2.1]hept-5-ene-2,3-dicarbonitrile (1), trans9,10-dihydro-9,10-ethanoanthracene-11,12-dicarbonitrile (2), and trans-bicyclo[2.2.1]hept-5-ene-2,3 dicarboxylic acid (13) are given in the Supplementary Material section.

\section{General synthesis of bis(4,5-dihydro-1H-imidazol-2-yl) derivatives 3 and 4}

Conventional synthesis (Method A). $6.94 \mathrm{mmol}$ of compound $\mathbf{1}$ (or compound 2) were dissolved in ethylenediamine $(50 \mathrm{~mL})$. The clear solution was heated with stirring in an argon atmosphere at $115^{\circ} \mathrm{C}$ for 24 hours. The resulting yellow color solution was cooled to $25^{\circ} \mathrm{C}$ and allowed to stand at this temperature for 24 hours. The occurred solid was filtered. The crude product was crystallized from benzene. Compound $\mathbf{3}$ was obtained as colorless powder $(1.21 \mathrm{~g}, 5.25 \mathrm{mmol})$ in $76 \%$ yield. Compound $\mathbf{3}$ was washed with chloroform and recrystallized from ethanol. Compound 4 was obtained $(1.69 \mathrm{~g}, 4.93 \mathrm{mmol}$ ) in $71 \%$ yield.

Microwave-assisted synthesis (Method B). $6.94 \mathrm{mmol}$ of compound 1 (or compound 2) were dissolved in ethylenediamine $(40 \mathrm{~mL})$. The solution was irradiated at atmospheric pressure using a mono-mode microwave device at $125{ }^{\circ} \mathrm{C}$ for 65 minutes with $250 \mathrm{~W}$ power. The resulting yellow color solution was cooled to room temperature and allowed to stand for 24 hours at this temperature. The occurred solid was filtered. The crude was crystallized from benzene. Compound 3 was obtained as colorless powder $(1.41 \mathrm{~g}, 6.12 \mathrm{mmol})$ in $88 \%$ yield, and compound 4 was isolated as colorless powder $(1.97 \mathrm{~g}, 5.76 \mathrm{mmol})$ in $83 \%$ yield.

trans-5,6-Bis(4,5-dihydro-1H-imidazol-2-yl)bicyclo[2.2.1]hept-2-ene (3). Mp: 206-208 ${ }^{\circ} \mathrm{C}$; IR $(\mathrm{KBr})\left(\mathrm{V}_{\max }, \mathrm{cm}^{-1}\right)$ : $3162,2973,2928,2862,1653,1604,1498,1470,1288,977 ;{ }^{1} \mathrm{H}$ NMR $\left(400 \mathrm{MHz} \mathrm{CDCl}_{3}\right): \delta_{\mathrm{H}}=6.37-6.35(1 \mathrm{H}, \mathrm{dd}$, J 3.2, $1.7 \mathrm{~Hz}), 6.13-6.11(1 \mathrm{H}, \mathrm{dd}$, J 3.0, $2.6 \mathrm{~Hz}), 4.14(2 \mathrm{H}, \mathrm{br}),. 3.64-3.62(4 \mathrm{H}, \mathrm{dd}, J 3.7,1.7 \mathrm{~Hz}), 3.59(4 \mathrm{H}, \mathrm{s}), 3.19-$ $3.16(2 \mathrm{H}, \mathrm{dd}, J 5.6,3.3 \mathrm{~Hz}), 3.14(1 \mathrm{H}, \mathrm{s}), 2.58-2.56(1 \mathrm{H}, \mathrm{d}, J 4.6 \mathrm{~Hz}), 1.75(1 \mathrm{H}, \mathrm{d}, \mathrm{A}$ part of AB system, J 8.6 Hz), 1.59 $(1 \mathrm{H}, \mathrm{dd}, \mathrm{B}$ part of $\mathrm{AB}$ system, $J$ 6.9, $1.7 \mathrm{~Hz}) ;{ }^{13} \mathrm{C} \mathrm{NMR}\left(100 \mathrm{MHz}, \mathrm{CDCl}_{3}\right): \delta_{\mathrm{C}}=170.2,169.4(\mathrm{C}=\mathrm{N}), 138.3,134.1(\mathrm{C}=\mathrm{C})$, 49.6, $49.4\left(\mathrm{CH}_{2}-\mathrm{N}\right), 48.4\left(\mathrm{CH}_{2}\right), 46.3,45.5$ (CH-bridgehead), 44.2, $43.6(\mathrm{CH})$; Anal. calc. for $\mathrm{C}_{13} \mathrm{H}_{18} \mathrm{~N}_{4}(230.31)$ : C, $67.80 ; \mathrm{H}, 7.88 ; \mathrm{N}, 24.33$; found C, 67.94; $\mathrm{H}, 8.06 ; \mathrm{N}, 24.57 \%$.

trans-11,12-Bis(4,5-dihydro-1H-imidazol-2-yl)-9,10-dihydro-9,10-ethanoanthracene (4). Mp: $282-284{ }^{\circ} \mathrm{C}$; IR $(\mathrm{KBr})\left(v_{\max }, \mathrm{cm}^{-1}\right): 3137,3074,3022,2936,2865,1612,1596,1496,1472,761 ;{ }^{1} \mathrm{H}$ NMR $\left(400 \mathrm{MHz}, \mathrm{DMSO}-d_{6}\right)$ : $\delta_{\mathrm{H}}=7.30-7.27(2 \mathrm{H}, \mathrm{m}), 7.22-7.20(2 \mathrm{H}, \mathrm{m}), 7.08-7.04(4 \mathrm{H}, \mathrm{m}), 4.66(2 \mathrm{H}, \mathrm{s}), 3.32-3.22(10 \mathrm{H}, \mathrm{m}) ;{ }^{13} \mathrm{C} \mathrm{NMR}(100$ $\left.\mathrm{MHz}, \mathrm{DMSO}-d_{6}\right): \delta_{\mathrm{C}}=168.4(\mathrm{C}=\mathrm{N}), 143.9,141.4(\mathrm{Ar} \mathrm{C}$ ipso), 126.0, 125.9, 125.2, $123.6(\mathrm{CH} \mathrm{Ar}), 46.94(\mathrm{CH}$ bridgehead), $42.70(\mathrm{CH})$; Anal. calc. for $\mathrm{C}_{22} \mathrm{H}_{22} \mathrm{~N}_{4}$ (342.44): C, 77.16; $\mathrm{H}, 6.48 ; \mathrm{N}, 16.36$; found C, 77.33; $\mathrm{H}, 6.68 ; \mathrm{N}$, $16.53 \%$.

trans-5,6-Bis[1-(2-chlorobenzoyl)-4,5-dihydro-1H-imidazol-2-yl]bicyclo[2.2.1]hept-2-ene (5). To a solution of compound $3(0.20 \mathrm{~g}, 0.87 \mathrm{mmol})$ in dry THF $(50 \mathrm{~mL})$, triethylamine $(0.24 \mathrm{~mL}, 1.74 \mathrm{mmol})$ was added at $0{ }^{\circ} \mathrm{C}$ and the mixture was stirred for 10 minutes. 2-Chlorobenzoyl chloride $(0.23 \mathrm{~mL}, 1.91 \mathrm{mmol})$ was added dropwise to the mixture and reflux maintained for 16 hours. The solvent was removed under reduced pressure. The residue was washed with $150 \mathrm{~mL}$ of water. After drying, the crude product was purified by column chromatography with a methanol / ethyl acetate $1: 1$ solvent mixture. The product 5 was obtained as colorless powder; yield: $0.38 \mathrm{~g}(0.75 \mathrm{mmol}, 86 \%)$; $\mathrm{mp}: 201-203{ }^{\circ} \mathrm{C}$; IR $(\mathrm{KBr})\left(v_{\max } \mathrm{cm}^{-1}\right): 3059,3019,2957,2925,2890$, $2861,1674,1645,1593,1439,1377,1221,767,703 ;{ }^{1} \mathrm{H} \mathrm{NMR}\left(400 \mathrm{MHz}, \mathrm{CDCl}_{3}\right): \delta_{\mathrm{H}} 7.58-7.56$ (2H, dd, J $6.2,0.9$ $\mathrm{Hz})$, 7.41-7.31 (6H, m, Ar-H), 6.23-6.19 (2H, m), 3.67-3.39 (8H, m), $3.15(1 \mathrm{H}, \mathrm{s}), 3.06(1 \mathrm{H}, \mathrm{s}), 3.04-3.02(1 \mathrm{H}, \mathrm{m})$, 
2.37-2.35 (1H, dd, J 4.0, $1.5 \mathrm{~Hz}), 1.71(1 \mathrm{H}, \mathrm{d}, \mathrm{A}$ part of $A B$ system, J 8.6 Hz), $1.53(1 \mathrm{H}, \mathrm{dd}, \mathrm{B}$ part of $A B$ system, J 7.1, 1.6 $\mathrm{Hz}) ;{ }^{13} \mathrm{C} N M R\left(100 \mathrm{MHz}, \mathrm{CDCl}_{3}\right): \delta_{\mathrm{C}}=175.8,174.6(\mathrm{C}=\mathrm{O}), 168.1,167.7(\mathrm{C}=\mathrm{N}), 137.6,135.2(\mathrm{C}=\mathrm{C}), 134.8,130.7$ $\left(\right.$ Ar- $\left.\mathrm{C}_{\text {ipso }}\right), 131.5,131.3,130.3,130.2,129.8,129.6,127.2,127.09\left(\mathrm{CH}_{\mathrm{Ar}}\right), 51.1,49.1,45.6,44.4(\mathrm{CH}), 48.3\left(\mathrm{CH}_{2}\right)$, 40.7, 40.36, 40.24, $39.72\left(\mathrm{~N}-\mathrm{CH}_{2}\right)$. Anal. calc. for $\mathrm{C}_{27} \mathrm{H}_{24} \mathrm{Cl}_{2} \mathrm{~N}_{4} \mathrm{O}_{2}(507.41): \mathrm{C}, 63.91 ; \mathrm{H}, 4.77 ; \mathrm{N}, 11.04 ;$ found $\mathrm{C}$, $64.12 ; H, 4.94 ; N, 11.22 \%$.

General synthesis of C-2 aromatic-heteroaromatic substituted imidazolines 6-10. Aromatic and heteroaromatic substituted imidazolines 6-10 were synthesized from the reaction of ethylenediamine and $\mathrm{N}$ bromosuccinimide with the different aromatic and heteroaromatic aldehydes. The synthetic method and detailed spectra of compounds 6-10 are given in the Supplementary Material section.

4,5-Dihydro-2-(3-thienyl)-1H-imidazole (10). Colorless powder; yield: 94\%; mp: $203-205{ }^{\circ} \mathrm{C}$; IR (ATR) ( $v_{\max }$ $\left.\mathrm{cm}^{-1}\right): 3113,3052,2927,2857,1600,1523,1497,1266,1177,981,679 ;{ }^{1} \mathrm{H}$ NMR $\left(400 \mathrm{MHz}, \mathrm{DMSO}-d_{6}\right): \delta_{\mathrm{H}}=$ $7.92(1 \mathrm{H}, \mathrm{s}), 7.58-7.56(1 \mathrm{H}, \mathrm{m}), 7.45,7.44(1 \mathrm{H}, \mathrm{d}, J 5.0 \mathrm{~Hz}), 6.86(1 \mathrm{H}, \mathrm{br}), 3.56(4 \mathrm{H}, \mathrm{s}) ;{ }^{13} \mathrm{C} \mathrm{NMR}(100 \mathrm{MHz}$, DMSO- $\left.d_{6}\right): \delta_{C}=161.8(\mathrm{C}=\mathrm{N}), 132.0\left(\right.$ Ar- $\left.\mathrm{C}_{\text {ipso }}\right), 126.6,126.3,125.9\left(\mathrm{CH}_{\mathrm{Ar}}\right), 48.8\left(\mathrm{CH}_{2}\right)$; Anal. calc. for $\mathrm{C}_{7} \mathrm{H}_{8} \mathrm{~N}_{2} \mathrm{~S}$ (152.22): C, 55.24; H, 5.30; N, 18.40; S, 21.07; found C, 55.46; H, 5.51; N, 18.63; S, $21.39 \%$.

trans-Bicyclo[2.2.1] hept-5-ene-2,3-dicarbonyl dichloride (14). Compound 13 (5.49 mmol) was dissolved in dry benzene $(25 \mathrm{~mL})$ and DMF $(0.1 \mathrm{~mL})$ and then oxalyl chloride $(1.02 \mathrm{mmol})$ rapidly added to the mixture. The mixture was refluxed for 4 hours until the $\mathrm{HCl}$ gas evolution had ceased. The gas evolved during the reaction was monitored by measuring the $\mathrm{pH}$ of the medium. After cooling the mixture to room temperature, the solvent was removed at reduced pressure. Anhydrous benzene $(10 \mathrm{~mL})$ was added to the crude product and stirred at room temperature for 10 minutes. The solvent was removed under reduced pressure. This process has been repeated three times. The light yellow liquid was isolated with $98 \%$ yield (1.18 g, $5.39 \mathrm{mmol})$. The product was used in the next step without purification. ${ }^{23,26}$

General synthesis of trans-5,6-bis[[4,5-dihydro-1H-imidazol-1-yl]carbonyl]bicyclo[2.2.1]hept-2-enes 15 and 16. To a solution of compound $7(3.28 \mathrm{mmol}$, or compound 10$)$, in dry THF (50 mL), triethylamine (3.66 mmol, $0.37 \mathrm{~g}, 0.51 \mathrm{~mL}$ ) was added and stirred at room temperature for 30 minutes. Compound 14 (0.36 g, 1.64 mmol) was added dropwise to the mixture at $0{ }^{\circ} \mathrm{C}$ and stirred at room temperature for 24 hours. The white solid which occurred at the end of the process was filtered. The solvent was removed in vacuum from the filtrate. The oily product was dissolved in methanol $(10 \mathrm{~mL})$ and precipitated in ice water $(200 \mathrm{~mL})$. The crude product was filtered and dried in vacuo. The colorless solid was crystallized with ethyl acetate.

trans-5,6-Bis[[4,5-dihydro-2-(3-thienyl)-1H-imidazol-1-yl]carbonyl]bicyclo[2.2.1]hept-2-ene (15). Colorless powder; yield: $0.50 \mathrm{~g}(1.11 \mathrm{mmol}, 68 \%) ; \mathrm{mp}: 198-200{ }^{\circ} \mathrm{C}$; IR (ATR) ( $\left.v_{\max } \mathrm{cm}^{-1}\right): 3110,3074,2980,2946,2869$, $1673,1614,1524,1358,1322,1266,1197,1007,710 ;{ }^{1} \mathrm{H}$ NMR $\left(400 \mathrm{MHz}, \mathrm{CDCl}_{3}\right): \delta_{\mathrm{H}}=7.50-7.40(3 \mathrm{H}, \mathrm{m}), 7.35$ $(1 \mathrm{H}, \mathrm{d}, J 2.8 \mathrm{~Hz}), 7.07(2 \mathrm{H}, \mathrm{ddd}, J 8.9,5.0,3.8 \mathrm{~Hz}), 6.36(1 \mathrm{H}, \mathrm{s}), 6.17(1 \mathrm{H}, \mathrm{dd}, J 5.5,2.7 \mathrm{~Hz}), 4.26-4.09(4 \mathrm{H}, \mathrm{m})$, 4.08-3.94 $(4 \mathrm{H}, \mathrm{m}), 3.81(1 \mathrm{H}, \mathrm{t}, J 3.9 \mathrm{~Hz}), 3.33(1 \mathrm{H}, \mathrm{s}), 3.20-3.07(2 \mathrm{H}, \mathrm{m}), 1.93(1 \mathrm{H}, \mathrm{d}, \mathrm{A}$ part of $\mathrm{AB}$ system, $J 8.7 \mathrm{~Hz})$, $1.56(1 \mathrm{H}, \mathrm{d}, \mathrm{B}$ part of $\mathrm{AB}$ system, $J .7 \mathrm{~Hz}) ;{ }^{13} \mathrm{C} \mathrm{NMR}\left(100 \mathrm{MHz}, \mathrm{CDCl}_{3}\right): \delta_{\mathrm{C}}=171.9,171.2(\mathrm{C}=\mathrm{O}), 155.1,154.7(\mathrm{C}=\mathrm{N})$, 137.9, $134.2(\mathrm{C}=\mathrm{C}), 133.2,133.0$ (thiophenyl- $\mathrm{C}_{\text {ipso }}$ ), 130.7, 130.6 (thiophenyl $\mathrm{CH}_{-5}$ ), 128.9 (thiophenyl $\mathrm{CH}_{-4}$ ), 127.0 (thiophenyl $\left.\mathrm{CH}_{-5}\right), 53.8,53.5$ ( $\mathrm{CH}_{2}$-imidazoline), 49.5, 48.67 ( $\mathrm{CH}$-bridgehead), 48.73, $48.6\left(\mathrm{CH}_{2}-\mathrm{N}\right), 47.4$ (bridge- $\left.\mathrm{CH}_{2}\right)$, 48.5, $45.9(\mathrm{O}=\mathrm{C}-\mathrm{CH})$; Anal. calc. for $\mathrm{C}_{23} \mathrm{H}_{22} \mathrm{~N}_{4} \mathrm{O}_{2} \mathrm{~S}_{2}(450.58)$ : C, 61.31; $\mathrm{H}, 4.92 ; \mathrm{N}, 12.43 ; \mathrm{S}, 14.23$; found $\mathrm{C}, 61.59$; $\mathrm{H}, 5.07 ; \mathrm{N}, 12.65 ; \mathrm{S}, 14.56 \%$.

trans-5,6-Bis[[2-(3-chlorophenyl)-4,5-dihydro-1H-imidazol-1-yl]carbonyl]bicyclo[2.2.1]hept-2-ene (16). Colorless powder; yield: $0.37 \mathrm{~g}(1.70 \mathrm{mmol}, 52 \%) ; \mathrm{mp}: 110-112{ }^{\circ} \mathrm{C}$; IR (ATR) $\left(\mathrm{v}_{\max }, \mathrm{cm}^{-1}\right): 3069,2975,2938$, $2872,1672,1595,1569,1358,1277,1261,1145,1000,794,753,704 ;{ }^{1} \mathrm{H} \mathrm{NMR}\left(400 \mathrm{MHz}, \mathrm{CDCl}_{3}\right): \delta_{\mathrm{H}}=7.49(1 \mathrm{H}$, s), $7.42(3 \mathrm{H}, \mathrm{ddd}, J$ 8.9, 6.1, $2.3 \mathrm{~Hz}), 7.40-7.29(4 \mathrm{H}, \mathrm{m}), 6.34(1 \mathrm{H}, \mathrm{d}, J 15.5 \mathrm{~Hz}), 6.12(1 \mathrm{H}, \mathrm{s}), 4.41-3.85(8 \mathrm{H}, \mathrm{m})$, 
$3.68(1 \mathrm{H}, \mathrm{t}, J 3.8 \mathrm{~Hz}), 3.30(1 \mathrm{H}, \mathrm{s}), 3.06(1 \mathrm{H}, \mathrm{s}), 2.98(1 \mathrm{H}, \mathrm{s}),,^{\prime} 1.83(1 \mathrm{H}, \mathrm{d}, \mathrm{A}$ part of AB system, J $7.8 \mathrm{~Hz}), 1.54(1 \mathrm{H}, \mathrm{d}$, B part of $A B$ system, $J 8.1 \mathrm{~Hz}) .{ }^{13} \mathrm{C} N M R\left(100 \mathrm{MHz}, \mathrm{CDCl}_{3}\right): \delta_{C}=171.6,170.9(\mathrm{C}=0), 159.5(\mathrm{C}=\mathrm{N}), 133.9,(\mathrm{CAr}$-ipso), 133.9, 133.7 (C=C), 130.2, 130.1, 129.2, 128.2, 126.2, $126.0(\mathrm{Ar} \mathrm{CH}), 54.4,54.0\left(\mathrm{CH}_{2}-\mathrm{N}\right), 49.0,48.1\left(\mathrm{CH}_{\text {bridgehead }}\right)$, 48.5, $45.8(\mathrm{O}=\mathrm{C}-\mathrm{CH}), 48.0,47.9\left(\mathrm{CH}_{2}-\mathrm{N}\right), 47.3,\left(\mathrm{CH}_{2}\right.$ bridge); Anal. calc. for $\mathrm{C}_{27} \mathrm{H}_{24} \mathrm{Cl}_{2} \mathrm{~N}_{4} \mathrm{O}_{2}(507.41): \mathrm{C}, 63.91 ; \mathrm{H}$, 4.77; N, 11.04; found C, 64.13; H, 4.83; N, 11.27\%.

General synthesis of $\mathbf{N}$-acyl substituted imidazolines with dibenzobarrelene skeleton 19-24. To a solution of the requisite imidazolines $(3.72 \mathrm{mmol}$, compounds 6, 7, 9-12) in dry THF (50 mL), triethylamine $(0.37 \mathrm{~g}, 3.66$ $\mathrm{mmol}, 0.51 \mathrm{~mL}$ ) was added, and then stirred at room temperature for 30 minutes. Compound 18 (0.57 g, 1.72 mmol) was added to this mixture at $0{ }^{\circ} \mathrm{C}$ and stirred at room temperature for 24 hours. The formed colorless solid was filtered. The solvent was removed in vacuo. The oily product was dissolved in methanol (10 $\mathrm{mL})$ and precipitated in ice-water $(200 \mathrm{~mL})$. The resulting colorless solid was filtered and dried at vacuum. The crude products were crystallized from THF, except for compound 23, crystallized from methanol.

trans-11,12-Bis[(4,5-dihydro-2-phenyl-1H-imidazol-1-yl)carbonyl]-9,10-dihydro-9,10-ethanoanthracene (19). Colorless powder, yield: $0.85 \mathrm{~g}(1.54 \mathrm{mmol}, 90 \%) ; \mathrm{mp}: 250-251{ }^{\circ} \mathrm{C}$; IR (ATR) $\left(\mathrm{v}_{\max }, \mathrm{cm}^{-1}\right): 3059,3026,2948$, $2866,1685,1625,1364,1273,1139,1023,767,691 ;{ }^{1} \mathrm{H}$ NMR $\left(400 \mathrm{MHz}, \mathrm{DMSO}-d_{6}\right): \delta_{\mathrm{H}}=7.47-7.40(4 \mathrm{H}, \mathrm{m})$, $7.38(2 \mathrm{H}, \mathrm{s}), 7.36-7.31(5 \mathrm{H}, \mathrm{m}), 7.28-7.22(4 \mathrm{H}, \mathrm{m}), 7.19-7.15(3 \mathrm{H}, \mathrm{m}), 4.85(2 \mathrm{H}, \mathrm{s}), 4.32(2 \mathrm{H}, \mathrm{dd}, J 19.3,9.7 \mathrm{~Hz})$, 4.11- $4.04(2 \mathrm{H}, \mathrm{m}), 4.03-3.96(2 \mathrm{H}, \mathrm{m}), 3.93-3.81(2 \mathrm{H}, \mathrm{m}), 3.49(2 \mathrm{H}, \mathrm{s}) ;{ }^{13} \mathrm{C} \mathrm{NMR}\left(100 \mathrm{MHz}, \mathrm{DMSO}-d_{6}\right): \delta_{\mathrm{c}}=$ $170.2(\mathrm{C}=\mathrm{O}), \quad 160.1(\mathrm{C}=\mathrm{N}), \quad 143.2,140.6$ (dibenzobarrelene-Cipso), 132.6 (CAr-ipso), 128.5, 127.8 (dibenzobarrelene-сн), 129.9, 128.8, 126.6, 126.2, 125.5, $123.8\left(\mathrm{CH}_{\text {phenyl }}\right), 54.5,48.1\left(\mathrm{CH}_{2}-\mathrm{N}\right), 48.5(\mathrm{CH}$-bridgehead), 46.1 (CH); Anal. calc. for $\mathrm{C}_{36} \mathrm{H}_{30} \mathrm{~N}_{4} \mathrm{O}_{2}$ (550.65): C, 78.52; $\mathrm{H}, 5.49 ; \mathrm{N}, 10.17$; found $\mathrm{C}, 78.73 ; \mathrm{H}, 5.66 ; \mathrm{N}, 10.39 \%$.

trans-11,12-Bis[[2-(3-chlorophenyl)-4,5-dihydro-1H-imidazol-1-yl]carbonyl]-9,10-dihydro-9,10-

ethanoanthracene (20). Colorless powder; yield: $0.86 \mathrm{~g}(1.40 \mathrm{mmol}, 75 \%) ; \mathrm{mp}: 241-243{ }^{\circ} \mathrm{C}$; IR (ATR) ( $V_{\max }$, $\left.\mathrm{cm}^{-1}\right)$ : 3073, 3026, 2964, 2867, 1730, 1680, 1629, 1459, 1355, 1261, 1143, 1000, 791, 762, 700, 560; ${ }^{1} \mathrm{H} \mathrm{NMR}$ $\left(400 \mathrm{MHz}, \mathrm{DMSO}-d_{6}\right): \delta_{\mathrm{H}}=7.49(2 \mathrm{H}, \mathrm{d}, \mathrm{J} 5.9 \mathrm{~Hz}), 7.45-7.39(4 \mathrm{H}, \mathrm{m}),, 7.31(4 \mathrm{H}, \mathrm{d}, J 9.5 \mathrm{~Hz}), 7.15(6 \mathrm{H}, \mathrm{dd}, J 12.5$, $4.9 \mathrm{~Hz}), 4.89(2 \mathrm{H}, \mathrm{s}), 4.35(2 \mathrm{H}, \mathrm{d}, \mathrm{J} 9.3 \mathrm{~Hz}),, 4.14-3.97(4 \mathrm{H}, \mathrm{m}),, 3.97-3.84(2 \mathrm{H}, \mathrm{m}), 3.55(1 \mathrm{H}, \mathrm{s}), 3.46(1 \mathrm{H}, \mathrm{s}) ;{ }^{13} \mathrm{C}$ NMR (100 MHz, DMSO- $\left.d_{6}\right): \delta_{C}=170.2(C=0), 158.9(C=N), 143.1,140.5$ (dibenzobarrelene- $\left.C_{i p s o}\right), 134.8,132.6$ (CAr-ipso), 130.0, 129.8, 128.2, 126.9, 126.7, 126.6, 125.4, 125.2, 124.0, 123.7 (Ar-cH), 54.7, 47.9 (CH $-\mathrm{N}), 52.5$, 48.3, 46.0, 45.9 (CH); Anal. calc. for $\mathrm{C}_{36} \mathrm{H}_{28} \mathrm{Cl}_{2} \mathrm{~N}_{4} \mathrm{O}_{2}$ (619.54): C, 69.79; $\mathrm{H}, 4.56 ; \mathrm{N}, 9.04$; found C, 69.81; $\mathrm{H}, 4.63$; N, $9.17 \%$.

trans-11,12-Bis[[4,5-dihydro-2-(2-thienyl)-1H-imidazol-1-yl]carbonyl]-9,10-dihydro-9,10-ethanoanthracene (21). Colorless powder; yield: $0.90 \mathrm{~g}(1.60 \mathrm{mmol}, 86 \%) ; \mathrm{mp}: 256-258{ }^{\circ} \mathrm{C}$; IR (ATR) $\left(\mathrm{V}_{\max }, \mathrm{cm}^{-1}\right): 3069,3034$, 2956, 2871, 1689, 1671, 1609, 1357, 1266, 1080, 681; ${ }^{1} \mathrm{H}$ NMR $\left(400 \mathrm{MHz}, \mathrm{DMSO}-d_{6}\right): \delta_{\mathrm{H}}=7.60(2 \mathrm{H}, \mathrm{dd}, J 4.5$, 1.7 Hz), $7.47(2 \mathrm{H}, \mathrm{d}, J 6.7 \mathrm{~Hz}), 7.43(1 \mathrm{H}, \mathrm{s}), 7.33(1 \mathrm{H}, \mathrm{s}), 7.23-7.12(4 \mathrm{H}, \mathrm{m}), 7.01-6.90(4 \mathrm{H}, \mathrm{m}), 4.85(2 \mathrm{H}, \mathrm{s}), 4.34$ $(2 \mathrm{H}, \mathrm{q}, J 9.8 \mathrm{~Hz}), 4.15(2 \mathrm{H}, \mathrm{td}, J$ 9.5, $4.7 \mathrm{~Hz}), 3.98(2 \mathrm{H}, \mathrm{ddd}, J 14.7,9.8,4.7 \mathrm{~Hz}), 3.84(2 \mathrm{H}, \mathrm{dt}, J 15.5,9.6 \mathrm{~Hz}), 3.64$ $(2 \mathrm{H}, \mathrm{s}) ;{ }^{13} \mathrm{C}$ NMR $\left(100 \mathrm{MHz}, \mathrm{DMSO}-d_{6}\right): \delta_{\mathrm{C}}=170.6(\mathrm{C}=\mathrm{O}), 154.6(\mathrm{C}=\mathrm{N}), 143.1,140.6$ (dibenzobarrelene- $\left.\mathrm{C}_{\mathrm{ipso}}\right)$, 133.7 (Cthiophene-ipso), 128.8, 127.2 (dibenzobarrelene-сн), 131.1, 129.4, 126.7, 126.3, 125.4, 123.9 ( $\left.\mathrm{CH}_{\text {thiophene }}\right), 54.0,48.7\left(\mathrm{CH}_{2}-\mathrm{N}\right), 49.0\left(\mathrm{CH}\right.$-bridgehead), $46.3(\mathrm{CH})$; Anal. calc. for: $\mathrm{C}_{32} \mathrm{H}_{26} \mathrm{~N}_{4} \mathrm{O}_{2} \mathrm{~S}_{2}(562.70): \mathrm{C}, 68.30 ; \mathrm{H}$, 4.66; N, 9.96; S, 11.40; found C, 68.53; H, 4.82; N, 10.11; S, 11.42\%.

trans-11,12-Bis[[4,5-dihydro-2-(3-thienyl)-1H-imidazol-1-yl]carbonyl]-9,10-dihydro-9,10-ethanoanthracene (22). Colorless powder; yield: $0.96 \mathrm{~g}(1.71 \mathrm{mmol}, 92 \%) ; \mathrm{mp}: 243-245{ }^{\circ} \mathrm{C}$; IR (ATR) $\left(\mathrm{V}_{\max }, \mathrm{cm}^{-1}\right): 3105,3026$, $2949,2862,1688,1679,1620,1457,1365,1259,1208,996,767,691,564 ;{ }^{1} \mathrm{H}$ NMR $\left(400 \mathrm{MHz}, \mathrm{DMSO}-d_{6}\right): \delta_{H}=$ $7.57(2 \mathrm{H}, \mathrm{s}), 7.47-7.41(2 \mathrm{H}, \mathrm{m}), 7.38(4 \mathrm{H}, \mathrm{dd}, J 5.1,2.8 \mathrm{~Hz}), 7.19-7.12(4 \mathrm{H}, \mathrm{m}), 7.02(2 \mathrm{H}, \mathrm{d}, J 4.9 \mathrm{~Hz}), 4.80(2 \mathrm{H}$, s), $4.29(2 \mathrm{H}, \mathrm{dd}, J 19.2,9.6 \mathrm{~Hz}), 4.06(2 \mathrm{H}, \mathrm{ddd}, J 15.1,10.2,5.3 \mathrm{~Hz}), 3.97(2 \mathrm{H}, \mathrm{td}, J 10.2,5.0 \mathrm{~Hz}), 3.91-3.81(2 \mathrm{H}$, m), $3.55(2 \mathrm{H}, \mathrm{s}) ;{ }^{13} \mathrm{C}$ NMR $\left(100 \mathrm{MHz}, \mathrm{DMSO}-d_{6}\right): \delta_{\mathrm{C}}=170.2(\mathrm{C}=\mathrm{O}), 155.5(\mathrm{C}=\mathrm{N}), 143.1,140.6$ (dibenzobarrelene- 


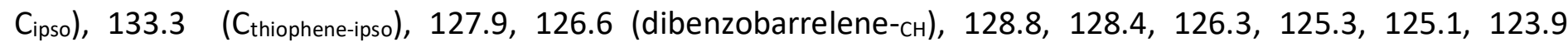

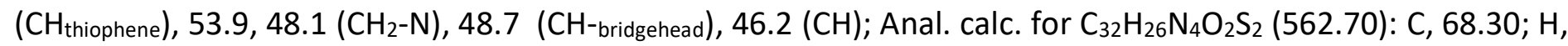
4.66; N, 9.96; S, 11.40; found C, 68.52; H, 4.79; N, 10.12; S, 11.58\%.

trans-11,12-Bis[(4,5-dihydro-2-sulfanyl-1H-imidazol-1-yl)carbonyl]-9,10-dihydro-9,10-ethanoanthracene (23). Colorless powder; yield: $0.52 \mathrm{~g}$ (1.13 mmol, 61\%); mp: 249-250 ${ }^{\circ} \mathrm{C}$; IR (ATR) $\left(\mathrm{v}_{\max }, \mathrm{cm}^{-1}\right): 3209,3030$, $2968,2894,1678,1532,1468,1349,1234,1208,1055,1011,765,755,608 ;{ }^{1} \mathrm{H}$ NMR $\left(400 \mathrm{MHz}, \mathrm{DMSO}-d_{6}\right): \delta_{\mathrm{H}}$ $=9.81(2 \mathrm{H}, \mathrm{s}), 7.32(2 \mathrm{H}, \mathrm{d}, J 7.1 \mathrm{~Hz}), 7.23(2 \mathrm{H}, \mathrm{d}, J 7.0 \mathrm{~Hz}), 7.14-7.02(4 \mathrm{H}, \mathrm{m}), 5.72(2 \mathrm{H}, \mathrm{s}), 4.90(2 \mathrm{H}, \mathrm{s}), 3.94$ $(2 \mathrm{H}, \mathrm{td}, J 10.9,8.4 \mathrm{~Hz}), 3.80(2 \mathrm{H}, \mathrm{td}, J 10.5,6.1 \mathrm{~Hz}), 3.58-3.43(4 \mathrm{H}, \mathrm{m}) ;{ }^{13} \mathrm{C} \mathrm{NMR}\left(100 \mathrm{MHz}, \mathrm{DMSO}-d_{6}\right): \delta_{\mathrm{c}}=$ $179.5(\mathrm{C}=\mathrm{O}), 173.0(\mathrm{C}=\mathrm{N}), 143.7,140.6$ (dibenzobarrelene- $\mathrm{C}_{\mathrm{ipso}}$ ), 126.2, 126.0, 124.9, 124.0 (dibenzobarreleneCHAr), 48.2 ( $\mathrm{CH}$-bridgehead), $48.0\left(\mathrm{CH}_{2}-\mathrm{N}\right), 46.8$ (dibenzobarrelene-cH); Anal. calc. for $\mathrm{C}_{24} \mathrm{H}_{22} \mathrm{~N}_{4} \mathrm{O}_{2} \mathrm{~S}_{2}$ (462.59): C, $62.31 ; \mathrm{H}, 4.79 ; \mathrm{N}, 12.11 ; \mathrm{S}, 13.86$; found $\mathrm{C}, 62.56 ; \mathrm{H}, 4.91 ; \mathrm{N}, 12.43 ; \mathrm{S}, 14.06 \%$.

trans-11,12-Bis[[4,5-dihydro-2-(methylsulfanyl)-1H-imidazol-1-yl]carbonyl]-9,10-dihydro-9,10-

ethanoanthracene (24). Colorless powder; yield: $0.57 \mathrm{~g}(1.15 \mathrm{mmol}, 62 \%) ; \mathrm{mp}: 260-262{ }^{\circ} \mathrm{C}$; IR (ATR) ( $v_{\max }$, $\left.\mathrm{cm}^{-1}\right)$ : 3072, 3037, 2958, 2925, 2885, 1666, 1588, 1458, 1411, 1371, 1190, 1004, 760, 746, 556; ${ }^{1} \mathrm{H}$ NMR (400 $\left.\mathrm{MHz} \mathrm{CDCl}_{3}\right): \delta_{\mathrm{H}}=7.34(2 \mathrm{H}, \mathrm{d}, J 6.8 \mathrm{~Hz}), 7.29-7.24(2 \mathrm{H}, \mathrm{m}), 7.18(4 \mathrm{H}, \mathrm{m}, J$ 7.6, $1.5 \mathrm{~Hz}), 4.48(2 \mathrm{H}, \mathrm{s}), 4.30-4.19$ $(2 \mathrm{H}, \mathrm{m}), 4.12-3.96(6 \mathrm{H}, \mathrm{m}), 3.56(2 \mathrm{H}, \mathrm{s}), 2.40(6 \mathrm{H}, \mathrm{s}) ;{ }^{13} \mathrm{C} \mathrm{NMR}\left(100 \mathrm{MHz}, \mathrm{CDCl}_{3}\right): \delta_{\mathrm{C}}=169.2(\mathrm{C}=0), 142.2,138.6$ (dibenzobarrelene- $\mathrm{C}_{\text {ipso }}$ ), 126.8, 126.7, 125.3, 122.7 (dibenzobarrelene- $\left.{ }_{-\mathrm{CH}}\right), 54.2,47.8\left(\mathrm{CH}_{2}-\mathrm{N}\right), 48.4(\mathrm{CH}-$ bridgehead), $47.1(\mathrm{CH}), 15.3\left(\mathrm{SCH}_{3}\right)$; Anal. calc. for $\mathrm{C}_{26} \mathrm{H}_{26} \mathrm{~N}_{4} \mathrm{O}_{2} \mathrm{~S}_{2}$ (490.64): C, 63.65; $\mathrm{H}, 5.34 ; \mathrm{N}, 11.42 ; \mathrm{S}, 13.07$; found $\mathrm{C}, 63.71 ; \mathrm{H}, 5.53 ; \mathrm{N}, 11.64 ; \mathrm{S}, 13.24 \%$.

General synthesis of 11,12-bis[(4,5-dihydro-1H-imidazol-1-yl)carbonyl]-9,10-dihydro-9,10-ethenoanthracenes 28-31 .To a solution of the requisite imidazoline (3.42 mmol, compounds 6, 8-10) in dry THF (50 $\mathrm{mL}), 3.76 \mathrm{mmol}$ of triethylamine $(0.38 \mathrm{~g}, 0.52 \mathrm{~mL})$ was added and stirred at room temperature for 30 minutes. $1.71 \mathrm{mmol}$ of compound $27(0.56 \mathrm{~g})$ was added to the mixture at $0{ }^{\circ} \mathrm{C}$ and then stirred at room temperature for 24 hours. The formed colorless solid was filtered. The solvent was removed in vacuum and the remaining solid was combined with the initially filtered solid and washed with water $(100 \mathrm{~mL})$. It was dried in vacuum. The crude products were crystallized with THF, and recrystallized from methanol / ethyl acetate 1:1 mixture. 11,12-Bis[(4,5-dihydro-2-phenyl-1H-imidazol-1-yl)carbonyl]-9,10-dihydro-9,10-ethenoanthracene (28). Colorless powder; yield: $0.84 \mathrm{~g}(1.54 \mathrm{mmol}, 90 \%) ; \mathrm{mp}: 238{ }^{\circ} \mathrm{C}$ (decomp.); IR (ATR) $\left(v_{\max }, \mathrm{cm}^{-1}\right): 3090,3061$, $2955,2869,1680,1621,1594,1559,1381,1354,695 ;{ }^{1} \mathrm{H}$ NMR $\left(400 \mathrm{MHz}, \mathrm{DMSO}-d_{6}\right): \delta_{\mathrm{H}}=8.01-7.98(2 \mathrm{H}, \mathrm{m}, \mathrm{J}$ 8, 1.6, $0.8 \mathrm{~Hz}), 7.64-7.58(3 \mathrm{H}, \mathrm{m}, J$ 7.6, 7.2, $1.2 \mathrm{~Hz}), 7.54-7.50(2 \mathrm{H}, \mathrm{m}, J$ 7.2, $1.2 \mathrm{~Hz}), 7.42-7.36(4 \mathrm{H}, \mathrm{m}, \mathrm{J} 4.8,3.6$ $\mathrm{Hz}), 7.32-7.30(1 \mathrm{H}, \mathrm{d}, J 7.6 \mathrm{~Hz}), 7.24-7.20(2 \mathrm{H}, \mathrm{t}, J 7.6 \mathrm{~Hz}), 7.00-6.97(4 \mathrm{H}, \mathrm{m}), 5.64(1 \mathrm{H}, \mathrm{s}), 5.38(1 \mathrm{H}, \mathrm{s}), 3.86(4 \mathrm{H}$, s), 3.69-3.65 (2H, t, J $8.8 \mathrm{~Hz}), 3.37-3.33(2 \mathrm{H}, \mathrm{t}, \mathrm{J} 8.8 \mathrm{~Hz}) ;{ }^{13} \mathrm{C} N M R\left(100 \mathrm{MHz}, \mathrm{DMSO}-d_{6}\right): \delta_{\mathrm{C}}=167.2,167.0(\mathrm{C}=0)$, $164.9(\mathrm{C}=\mathrm{N}), 159.3(\mathrm{C}=\mathrm{C}), 146.1,145.6$ (dibenzobarrelene- $\left.\mathrm{C}_{\text {Ar-ipso }}\right), 132.4$ ( $\left.\mathrm{C}_{\text {Ar-ipso }}\right), 133.6,129.9,129.4,128.8$, 128.7, $127.6\left(\mathrm{CH}_{\mathrm{Ar}}\right), 125.1,125.0,123.9,123.7$ (dibenzobarrelene- $\mathrm{CHAr}$ ), 52.9, 52.0 (dibenzobarrelene- $\mathrm{CH}-$ bridgehead), 53.7, 48.3, $46.3\left(\mathrm{CH}_{2}-\mathrm{N}\right)$; Anal. calc. for $\mathrm{C}_{36} \mathrm{H}_{28} \mathrm{~N}_{4} \mathrm{O}_{2}$ (548.63): C, 78.81; $\mathrm{H}, 5.14 ; \mathrm{N}, 10.21$; found C, 78.06; H, 5.22; N, 10.44\%.

\section{1,12-Bis[[4,5-dihydro-2-(4-methoxyphenyl)-1H-imidazol-1-yl]carbonyl]-9,10-dihydro-9,10-}

ethenoanthracene (29). Colorless powder; yield: $0.81 \mathrm{~g}$ (1.33 mmol, 78\%); mp: 238-240 ${ }^{\circ} \mathrm{C}$; IR (ATR) ( $v_{\max }$ $\left.\mathrm{cm}^{-1}\right)$ : 3067, 2961, 2921, 2852, 1657, 1605, 1511, 1458, 1353, 1252, 1173, 1013, 837, 608; ${ }^{1} \mathrm{H} \mathrm{NMR}(400 \mathrm{MHz}$, DMSO- $\left.d_{6}\right): \delta_{H}=7.50(4 \mathrm{H}, \mathrm{d}, J 8.8 \mathrm{~Hz}), 7.36(4 \mathrm{H}, \mathrm{dd}, J 5.3,3.2 \mathrm{~Hz}), 7.06(4 \mathrm{H}, \mathrm{dd}, J 5.3,3.2 \mathrm{~Hz}), 6.76(4 \mathrm{H}, \mathrm{d}, 3 J 8.8$ $\mathrm{Hz}), 5.34(\mathrm{~s}, 2 \mathrm{H}), 3.89-3.83(4 \mathrm{H}, \mathrm{t}, J 8.3 \mathrm{~Hz}), 3.79(\mathrm{~s}, 6 \mathrm{H}), 3.65(4 \mathrm{H}, \mathrm{t}, J 8.3 \mathrm{~Hz}) ;{ }^{13} \mathrm{C} \mathrm{NMR}\left(100 \mathrm{MHz}, \mathrm{DMSO}-d_{6}\right): \delta_{\mathrm{C}}$ $=165.6(\mathrm{C}=\mathrm{O}), 161.4\left(\mathrm{CH}_{3} \mathrm{O}-\mathrm{C}_{\text {Ar-ipso }}\right), 159.4(\mathrm{C}=\mathrm{N}), 147.6(\mathrm{C}=\mathrm{C}), 143.6$ (dibenzobarrelene- $\left.\mathrm{C}_{\text {Ar-ipso }}\right), 129.9,125.6$ (dibenzobarrelene-CHAr), $123.7\left(\mathrm{CH}_{\mathrm{Ar}}\right), 123.0\left(\mathrm{C}_{\mathrm{Ar}-\mathrm{ips}}\right), 113.4\left(\mathrm{CH}_{\mathrm{Ar}}\right), 55.2$ (dibenzobarrelene-CH-bridgehead), 54.0, 
49.1 ( $\mathrm{CH}_{2}$-imidazoline); $53.2\left(\mathrm{OCH}_{3}\right)$, Anal. calc. for $\mathrm{C}_{38} \mathrm{H}_{32} \mathrm{~N}_{4} \mathrm{O}_{4}(608.69)$ : C, 74.98; $\mathrm{H}, 5.30 ; \mathrm{N}, 9.20$; found $\mathrm{C}$, 75.12; H, 5.59; N, 9.46\%.

11,12-Bis[[4,5-dihydro-2-(2-thienyl)-1H-imidazol-1-yl]carbonyl]-9,10-dihydro-9,10-ethenoanthracene (30). Colorless powder; yield: $0.82 \mathrm{~g}(1.47 \mathrm{mmol}, 86 \%) ; \mathrm{mp}: 234-236{ }^{\circ} \mathrm{C}$; IR (ATR) $\left(v_{\max }, \mathrm{cm}^{-1}\right): 3072,3058,2973$, $2901,2873,1677,1662,1620,1558,1378,1357,1290,1005,715,613 ;{ }^{1} \mathrm{H}$ NMR $\left(400 \mathrm{MHz}, \mathrm{DMSO}-d_{6}\right): \delta_{\mathrm{H}}=$ 7.83-7.81 (2H, d, J $10 \mathrm{~Hz}), 7.57-7.47(2 \mathrm{H}, \mathrm{m}), 7.47-7.35(4 \mathrm{H}, \mathrm{m}), 7.17(1 \mathrm{H}, \mathrm{t}, J 4.1 \mathrm{~Hz}), 7.07-6.96(4 \mathrm{H}, \mathrm{m}), 6.85$ $(1 \mathrm{H}, \mathrm{s}), 5.64(1 \mathrm{H}, \mathrm{s}), 5.49(1 \mathrm{H}, \mathrm{s}), 3.73(4 \mathrm{H}, \mathrm{s}), 3.70-3.63(2 \mathrm{H}, \mathrm{t}, J 8.0 \mathrm{~Hz}), 3.41(2 \mathrm{H}, \mathrm{t}, J 8.0 \mathrm{~Hz}) ;{ }^{13} \mathrm{C} \mathrm{NMR}(100$ $\left.\mathrm{MHz}, \mathrm{DMSO}-d_{6}\right): \delta_{\mathrm{C}}=167.0,166.2(\mathrm{C}=\mathrm{O}), 159.4(\mathrm{C}=\mathrm{N}), 153.6(\mathrm{C}=\mathrm{C}), 145.7,145.2$ (dibenzobarrelene-cAr-ipso), 133.0 (C Ar-ipso), 131.9, 129.5, $128.6\left(\mathrm{CH}_{\mathrm{Ar}}\right), 127.3,125.3,125.2,123.9$ (dibenzobarrelene-CHAr), 53.3, 53.1 (dibenzobarrelene-cHbridgehead), 51.4, 48.8, $47.7\left(\mathrm{CH}_{2}-\mathrm{N}\right)$. Anal. calc. for $\mathrm{C}_{32} \mathrm{H}_{24} \mathrm{~N}_{4} \mathrm{O}_{2} \mathrm{~S}_{2}$ (560.69): $\mathrm{C}, 68.55 ; \mathrm{H}, 4.31$; N, 9.99; S, 11.44; found C, 68.73; H, 4.53; N, 10.15; S, $11.67 \%$.

11,12-Bis[[4,5-dihydro-2-(3-thienyl)-1H-imidazol-1-yl]carbonyl]-9,10-dihydro-9,10-ethenoanthracene (31). Colorless powder; yield: $0.88 \mathrm{~g}(1.57 \mathrm{mmol}, 92 \%) ; \mathrm{mp}: 138-139{ }^{\circ} \mathrm{C}$; IR (ATR) $\left(v_{\max }, \mathrm{cm}^{-1}\right): 3096,2985,2977$, $2925,2903,2857,1705,1619,1602,1576,1376,1286,669,624 ;{ }^{1} \mathrm{H}$ NMR $\left(400 \mathrm{MHz}, \mathrm{DMSO}-d_{6}\right): \delta_{\mathrm{H}}=8.71(1 \mathrm{H}$, d, J $1.5 \mathrm{~Hz}), 7.85(1 \mathrm{H}, \mathrm{d}, J 1.7 \mathrm{~Hz}), 7.80-7.67(2 \mathrm{H}, \mathrm{m}), 7.45-7.28(6 \mathrm{H}, \mathrm{m}), 6.99(4 \mathrm{H}, \mathrm{dd}, J 5.0,3.3 \mathrm{~Hz}), 5.66(1 \mathrm{H}$, s), $5.39(1 \mathrm{H}, \mathrm{s}), 3.83(4 \mathrm{H}, \mathrm{s}), 3.63(2 \mathrm{H}, \mathrm{t}, J 8.4 \mathrm{~Hz}), 3.33(2 \mathrm{H}, \mathrm{t}, J 8.5 \mathrm{~Hz}) ;{ }^{13} \mathrm{C} \mathrm{NMR}\left(100 \mathrm{MHz}, \mathrm{DMSO}-d_{6}\right): \delta_{\mathrm{c}}=$ 167.7, 167.5 (C=O), 159.9 (C=N), 154.9 (C=C), 146.3, 145.7 (dibenzobarrelene-cAr-ipso), 133.1 (CAr-ipso), 129.1, 128.8, 128.4, 127.1, $\left(\mathrm{CH}_{\mathrm{Ar}}\right), 125.1,124.9,123.8,123.7$ (dibenzobarrelene-CHAr), 53.3, 52.9 (dibenzobarrelene- $\mathrm{CH}$ bridgehead), 52.1, 48.2, 45.5, $45.4\left(\mathrm{CH}_{2}-\mathrm{N}\right)$. Anal. calc. for $\mathrm{C}_{32} \mathrm{H}_{24} \mathrm{~N}_{4} \mathrm{O}_{2} \mathrm{~S}_{2}$ (560.69): C, 68.55; $\mathrm{H}, 4.31 ; \mathrm{N}, 9.99 ; \mathrm{S}$, 11.44; found C, 68.73; H, 4.43; N, 10.18; S, 11.62\%.

\section{Acknowledgments}

We are indebted to the Firat University Research Project Coordination Unit (Project Number FF.13.04 and FF.17.17) for financial support of this work. We would like to thank Assos. Prof. Dr. Bünyamin Çoşut for contributions to the interpretation of X-ray analyses.

\section{Supplementary Material}

The synthesis of compounds $\mathbf{1}, \mathbf{2}, \mathbf{6 - 1 0}, 13$ and the copies of IR, ${ }^{1} \mathrm{H}$ NMR, ${ }^{13} \mathrm{C}$ NMR spectra of compounds 3-10, 15, 16, 19-24, 28-31 presented in the Supplementary Material.

\section{References}

1. Saczewski, F.; Kornicka, A.; Balewski, L. Expert Opin. Ther. Pat. 2016, 26, 1031. https://doi.org/10.1080/13543776.2016.1210128

2. Veale, C. G.; Davies-Coleman, M. T. Alkaloids Chem. Biol. 2014, 73, 1. https://doi.org/10.1016/B978-0-12-411565-1.00001-9

3. Vassilev, L. T.; Vu, B. T.; Graves, B.; CarvaJal, D.; Podlaski, F.; Filipovic, Z.; Kong, N.; Kammlott, U.; Lukacs, C.; Klein, C.; Fotouhi, N.; Liu, E. A. Science 2004, 303, 844.

https://doi.org/10.1126/science.1092472 
Arkivoc 2018, part vii, 0-0

4. Krasavin, M. Eur. J. Med. Chem. 2015, 97, 525. https://doi.org/10.1016/J.eJmech.2014.11.028

5. Crouch, R. D. Tetrahedron 2009, 65, 2387. https://doi.org/10.1016/J.tet.2008.12.022

6. Servi, S.; Genc, M.; Gur, S.; Koca, M. Eur. J. Med. Chem. 2005, 40, 687. https://doi.org/10.1016/J.eJmech.2005.02.002

7. Nasr-Esfahani, M.; Montazerozohori ,M.; Moghadam, M.; Akhlaghi, P. Arkivoc 2010, (ii), 97. https://doi.org/10.3998/ark.5550190.0011.207

8. Genc, M.; Servi, S. Synth. Commun. 2009, 39, 3263. https://doi.org/10.1080/00397910902739896

9. Mirkhani, V.; Moghadam, M.; TangestanineJad, S.; Kargar, H. Tetrahedron Lett. 2006, 47, 2129. https://doi.org/10.1016/J.tetlet.2006.01.127

10. Pathan, M. Y.; Paike, V. V.; Pachmase, P. R.; More, S. P.; Ardhapure, S. S.; Pawar, R. P. Arkivoc 2006, (xv), 205.

https://doi.org/10.3998/ark.5550190.0007.f25

11. Servi, S.; Genc, M. Synth. Commun. 2007, 37, 3173.

https://doi.org/10.1080/00397910701545320

12. Braddock, D. C.; Cailleau, T.; Cansell, G.; Hermitage, S. A.; Pouwer, R. H.; Redmond, J. M.; White, A. J. P. Tetrahedron: Asymmetry 2010, 21, 2911.

https://doi.org/10.1016/J.tetasy.2010.12.002

13. Zhu, Q.; Lu, Y. Org. Lett. 2010, 12, 4156.

https://doi.org/10.1021/ol101747n

14. Ma, K.; You, J. Chem. Eur. J. 2007, 13, 1863.

https://doi.org/10.1002/chem.200601220

15. Merino, P.; Marqués-López, E.; TeJero, T.; Herrera, R. P. Synthesis 2010, 1. https://doi.org/10.1055/s-0029-1217130

16. Murai, K.; Fukushima, S.; Hayashi, S.; Takahara, Y.; FuJioka, H. Org. Lett. 2010, 12, 964. https://doi.org/10.1021/ol902958m

17. Weatherwax, A.; Abraham, C. J.; Lectka, T. Org. Lett. 2005, 7, 3461. https://doi.org/10.1021/ol0511070

18. Akalay, D.; Dürner, G.; Bats, J. W.; Bolte, M.; Göbel, M. W. J. Org. Chem. 2007, 72, 5618. https://doi.org/10.1021/Jo070534J

19. Basavaiah, D.; Reddy, B. S.; Badsara, S. S. Chem. Rev. 2010, 110, 5447. https://doi.org/10.1021/cr900291g

20. Ishii, A.; Shibata, M.; Nakata, N. Bull. Chem. Soc. Jpn. 2016, 89, 1470. https://doi.org/10.1246/bcsJ.20160287

21. Lesyk, R.; Zimenkovsky, B.; Atamanyuk, D.; Jensen, F.; Kieć-Kononowicz, K.; Gzella, A. Bioorg. Med. Chem. 2006, 14, 5230. https://doi.org/10.1016/J.bmc.2006.03.053

22. Qin, Q.-P.; Liu, Y.-C.; Wang, H.-L.; Qin, J.-L.; Cheng, F.-J.; Tang, S.-F.; Liang, H. Metallomics 2015, 7, 1124. https://doi.org/10.1039/c5mt00027k

23. Çapan, I.; Servi, S. Synth. Commun. 2018, 48, 1164. https://doi.org/10.1080/00397911.2018.1437449

24. Mowry, D. T. J. Am. Chem. Soc. 1947, 69, 573. https://doi.org/10.1021/Ja01195a032 
25. Takacs, J. M.; Quincy, D. A.; Shay, W.; Jones, B. E.; Ross, C. R. Tetrahedron: Asymmetry 1997, 8, 3079. https://doi.org/10.1016/S0957-4166(97)00323-6

26. Alibert, S.; Santelli-Rouvier, C.; Pradines, B.; Houdoin, C.; Parzy, D.; Karolak-WoJciechowska, J.; Barbe, J. J. Med. Chem. 2002, 45, 3195.

https://doi.org/10.1021/Jm011046I

27. Tydlitát, J.; Bureš, F.; Kulhánek, J.; Mloston, G.; Růžička, A. Tetrahedron: Asymmetry 2012, $23,1010$. https://doi.org/10.1016/J.tetasy.2012.06.016

28. Dalko, P. I.; Brun, V.; Langlois, Y. Tetrahedron Lett. 1998, 39, 8979.

https://doi.org/10.1016/S0040-4039(98)02032-2

29. Mitchell, J. M.; Finney, N. S. Tetrahedron Lett. 2000, 41, 8431.

https://doi.org/10.1016/s0040-4039(00)01501-x

30. Zhang, J.; Wang, X.; Yang, M.; Wan, K.; Yin, B.; Wang, Y.; Li, J.; Shi, Z. Tetrahedron Lett. 2011, $52,1578$. https://doi.org/10.1016/J.tetlet.2011.01.082

31. FuJioka, H.; Murai, K.; Ohba, Y.; Hiramatsu, A.; Kita, Y. Tetrahedron Lett. 2005, 46, 2197. https://doi.org/10.1016/J.tetlet.2005.02.025

32. FuJioka, H.; Murai, K.; Kubo, O.; Ohba, Y.; Kita, Y. Tetrahedron 2007, 63, 638. https://doi.org/10.1016/J.tet.2006.11.007

33. Neef, G.; Eder, U.; Sauer, G. J. Org. Chem. 1981, 46, 2824. https://doi.org/10.1021/Jo00326a055

34. Ren, Y.-M.; Cai, C. Org. Prep. Proced. Int. 2008, 40, 101. https://doi.org/10.1080/00304940809356644

35. Sun, M.; Wei, H.-T.; Li, D.; Zheng, Y.-G.; Cai, J. Synth. Commun. 2008, 38, 1351. 